\title{
Successful Treatment of Severe Myocardial Injury Complicated With Refractory Cardiogenic Shock Caused by AOPP Using Extracorporeal Membrane Oxygenation
}

\author{
Yang Li \\ Department of Emergency Medicine \\ ChunShui Cao \\ Department of Emergency Medicine \\ XiaoLong Luo \\ Department of Emergency Medicine \\ Liang Huang ( $\sim 919584208 @ q q . c o m$ ) \\ First Affiliated Hospital of Nanchang University
}

\section{Case report}

Keywords: AOPP, VA-ECMO, ECMO

Posted Date: November 13th, 2020

DOl: https://doi.org/10.21203/rs.3.rs-104375/v1

License: (c) (i) This work is licensed under a Creative Commons Attribution 4.0 International License.

Read Full License 


\section{Abstract}

Acute organophosphorus pesticide poisoning (AOPP) is a common critical illness observed in clinical practice, and severe AOPP can cause serious cardiac toxicity. We report a case of severe myocardial injury caused by AOPP complicated with refractory cardiogenic shock that was successfully rescued with veno-arterial extracorporeal membrane oxygenation (VA-ECMO) therapy. We suggest that for patients with severe myocardial injury complicated with cardiogenic shock following AOPP, the use of VA-ECMO therapy can improve the prognosis.

\section{Introduction}

Acute organophosphorus pesticide poisoning (AOPP) is one of the most common critical illnesses observed in the emergency department. Annually, approximately 200,000 persons die from AOPP worldwide, and the mortality rate is generally above $15 \%^{[1,2]}$. AOPP can cause damage to the functions of the heart, lungs, brain and other systemic organs. Among them, cardiotoxicity mainly manifests as myocardial congestion, interstitial edema, ischemia and reversible interstitial inflammation ${ }^{[3]}$. However, when AOPP causes severe myocardial injury with refractory cardiogenic shock, the mortality rate exceeds $60 \%[4,5]$.

The cardiac toxicity caused by AOPP is mostly reversible. Extracorporeal membrane oxygenation (ECMO) can help patients through the critical period and lead to a better prognosis. For patients with severe myocardial injury who do not respond to traditional supportive care, the early application of ECMO is indicated ${ }^{[6-8]}$. We report a case of a middle-aged woman who manifested extensive myocardial injury and a life-threatening hemodynamic disorder after the oral consumption of approximately $300 \mathrm{ml}$ of organophosphorus pesticide. After conventional therapy failed, VA-ECMO was initiated.

\section{Case Report}

A 43-year-old woman was admitted to the emergency department with acute respiratory failure and hypotension 13 hours after the oral consumption of $300 \mathrm{ml}$ of phoxim pesticide. She had no history of heart disease. The physical examination on admission showed the following: temperature, $36.8^{\circ} \mathrm{C}$; heart rate, 84 beats/min; respiratory rate, 17 beats/min; and blood pressure, 81/42 $\mathrm{mm} \mathrm{Hg}$ (norepinephrine, $0.2 \mu \mathrm{g} / \mathrm{kg} / \mathrm{min}$ ). She had clear consciousness, the pupils on both sides were equal and $3.5 \mathrm{~mm}$ in diameter, the skin was dry all over the body, there were no wet rales over both lungs on pulmonary auscultation, and there was no murmur on heart auscultation. The arterial blood gas analysis and myocardial enzyme spectrum were normal, while electrocardiography (ECG) showed sinus tachycardia on admission. After admission, the patient was intubated and mechanically ventilated, analgesia and sedation were administered, rapid fluid expansion combined with norepinephrine was administered as an anti-shock treatment, hemoperfusion was performed, and atropine was administered to reverse the inhibitory effects of AOPP on cholinesterase. 
Twenty-three hours after admission, the ECG (Fig. 1A) showed extensive lead ST segment elevation. The patient progressively deteriorated over the 36 hours after admission, and the ECG showed ventricular arrhythmia with abnormal Q wave and ST-T segment changes in some leads (Fig. 1B), which indicated severe myocardial injury. Echocardiography revealed an ejection fraction of $30 \%$. The laboratory results showed a notable cTnl level of $11.0 \mu \mathrm{g} / \mathrm{L}$ (reference range, 0.01-0.023); the NT-ProBNP level was $26700 \mathrm{ng} / \mathrm{L}$ (reference range, 300-450), the creatine kinase level was $2454 \mathrm{U} / \mathrm{L}$ (reference range, 40200 ), and the creatine kinase-MB isoenzyme level was $166 \mathrm{U} / \mathrm{L}$ (reference range, $0-24$ ). She subsequently developed cardiogenic shock due to severe myocardial injury with complications of ventricular arrhythmia; thus, VA-ECMO was immediately initiated.

Three hours after the initiation of ECMO, the ECG showed a decrease in the ST segment (Fig. 1C). Her hemodynamics improved 6 hours after the initiation of ECMO. On the 3rd day after the initiation of ECMO, the myocardial enzyme indicators decreased significantly, and the left ventricular ejection fraction was $46 \%$. VA-ECMO was withdrawn. On the 11 th day after admission, she was transferred out of the intensive care unit, and at the 25-day follow-up, the patient had recovered and was discharged.

\section{Discussion}

AOPP often causes severe cardiac toxicity. Chen ${ }^{[2]}$ et al found that $52.0 \%$ of AOPP patients developed acute myocardial injury. The main mechanisms underlying myocardial injury may be as follows: 1) direct toxic effects on the myocardium by pesticides, solvents, impurities, etc.; 2) sympathetic and parasympathetic dysfunction, the release of a large quantity of catecholamines and increased sensitivity of the heart to catecholamines, causing coronary artery spasm, myocardial ischemia and myocardial injury; 3 ) the accumulation of a large amount of acetylcholine and the release of a large quantity of cytokines and inflammatory mediators affecting myocardial cells and resulting in toxic myocarditis; and 4) complications of respiratory failure, severe electrolyte disturbances, and acidosis causing internal environmental disturbances and aggravating myocardial damage ${ }^{[3,9,10]}$. In addition, Anand ${ }^{[11]}$ et al found that the pathology of myocardial injury in patients with AOPP was characterized by myocardial interstitial edema, vascular congestion and inflammatory changes in the myocardium. Severe myocardial injury due to AOPP often leads to various life-threatening cardiac arrhythmias, which are the main cause of sudden death.

As a bridge to recovery from cardiopulmonary failure, ECMO can improve the survival rate of patients with refractory cardiogenic shock following poisoning ${ }^{[12-14]}$. Vardon ${ }^{[15]}$ et al. reported the successful rescue of patients with intractable ventricular arrhythmia and cardiogenic shock caused by severe yew poisoning using VA-ECMO. In addition, ECMO was used in patients with severe aluminum phosphide poisoning, reducing the mortality rate from $84.4-40 \%{ }^{[5]}$. In addition, in a retrospective cohort analysis of persistent cardiac arrest or severe shock caused by drug poisoning, Masson [16] et al. found that the survival rate of patients who received ECMO was as high as $86 \%$, while that of patients who did not receive ECMO was only $48 \%$. Furthermore, in a meta-analysis of the application of ECMO in patients with 
severe cardiac damage caused by drug poisoning, the survival rate was as high as $66 \%{ }^{[17]}$. Thus, ECMO can be used as an effective therapeutic option for patients with severe poisoning.

The successful use of ECMO in patients with severe cardiotoxicity due to poisoning with various drugs has been reported. However, reports of the utilization of ECMO in patients with cardiogenic shock caused by AOPP are rare. We used ECMO to successfully rescue a patient with severe myocardial injury caused by AOPP associated with refractory cardiogenic shock and fatal arrhythmia. ECMO serves as a bridge to recovery in patients with severe poisoning, allowing the injured myocardium to rest. With the removal of the organophosphorus pesticides, cardiac function can be restored by the application of ECMO. Therefore, we recommend that for patients with refractory cardiogenic shock caused by AOPP, ECMO should be implemented early after the failure of conventional therapy ${ }^{[18,19]}$.

\section{Conclusions}

AOPP can cause fatal cardiac injury, and when the patient does not respond to conventional therapy, the early use of ECMO is an effective therapeutic option for refractory cardiogenic shock caused by AOPP.

\section{Declarations}

\section{Funding statement}

This work was supported by the Department of Education Science and Technology Research Project of Jiangxi Province of China (170119).

\section{Author contribution statement}

Yang Li, ChunShui Cao and XiaoLong Luo performed the initial literature review, data analysis, and wrote the manuscript. Liang Huang assisted in analyzing the case and critically revised the manuscript to the final form. All authors approved the final version for submission.

\section{Consent}

Written informed consent was obtained from the patient for publication of this case report. A copy of the written consent is available for review by the Editor-in-Chief of this journal on request.

\section{Declaration of competing interest}

The authors declare that they have no known competing financial interests or personal relationships that could have appeared to influence the work reported in this paper.

\section{Disclosure statement}

No potential conflict of interest was reported by the authors. 


\section{References}

1. Tang W, Ruan F, Chen Q, et al. Independent Prognostic Factors for Acute Organophosphorus Pesticide Poisoning[J]. Respir Care. 2016;61(7):965-70.

2. Chen KX, Zhou XH, Sun CA, et al. Manifestations of and risk factors for acute myocardial injury after acute organophosphorus pesticide poisoning[J]. Medicine. 2019;98(6):e14371.

3. He X, Li C, Wei D, et al. Cardiac abnormalities in severe acute dichlorvos poisoning[J]. Crit Care Med. 2011;39(8):1906-12.

4. De Lange DW, Sikma MA, Meulenbelt J. Extracorporeal membrane oxygenation in the treatment of poisoned patients[J]. Clin Toxicol (Phila). 2013;51(5):385-93.

5. Mohan B, Gupta V, Ralhan S, et al. Impact of extra-corporeal membrane oxygenation on outcome of aluminium phosphide poisoning complicated with myocardial dysfunction[J]. Clin Toxicol (Phila). 2019;57(11):1095-102.

6. Sun T, Guy A, Sidhu A, et al. Veno-arterial extracorporeal membrane oxygenation (VA-ECMO) for emergency cardiac support[J]. J Crit Care. 2018;44:31-8.

7. Megarbane B, Leprince P, Deye $\mathrm{N}$, et al. Emergency feasibility in medical intensive care unit of extracorporeal life support for refractory cardiac arrest[J]. Intensive Care Med. 2007;33(5):758-64.

8. Pozzi M, Koffel C, Djaref C, et al. High rate of arterial complications in patients supported with extracorporeal life support for drug intoxication-induced refractory cardiogenic shock or cardiac arrest[J]. J Thorac Dis. 2017;9(7):1988-96.

9. Georgiadis N, Tsarouhas K, Tsitsimpikou C, et al. Pesticides and cardiotoxicity. Where do we stand? [J]. Toxicol Appl Pharmacol. 2018;353:1-14.

10. Wang $X$, Martinez MA, Dai M, et al. Permethrin-induced oxidative stress and toxicity and metabolism. A review[J]. Environ Res. 2016;149:86-104.

11. Anand S, Singh S, Nahar Saikia U, et al. Cardiac abnormalities in acute organophosphate poisoning[J]. Clin Toxicol (Phila). 2009;47(3):230-5.

12. Wang D, Zhang G, Zhang W, et al. Successful extracorporeal membrane oxygenation support for severe acute diquat and glyphosate poisoning: A case report[J]. Medicine. 2019;98(6):e14414.

13. Maskell KF, Ferguson NM, Bain J, et al. Survival After Cardiac Arrest: ECMO Rescue Therapy After Amlodipine and Metoprolol Overdose[J]. Cardiovasc Toxicol. 2017;17(2):223-5.

14. St-Onge M, Anseeuw K, Cantrell FL, et al. Experts Consensus Recommendations for the Management of Calcium Channel Blocker Poisoning in Adults[J]. Crit Care Med. 2017;45(3):e306-15.

15. Vardon Bounes F, Tardif E, Ruiz S, et al. Suicide attempt with self-made Taxus baccata leaf capsules: survival following the application of extracorporeal membrane oxygenation for ventricular arrythmia and refractory cardiogenic shock[J]. Clin Toxicol (Phila). 2017;55(8):925-8.

16. Masson R, Colas V, Parienti JJ, et al. A comparison of survival with and without extracorporeal life support treatment for severe poisoning due to drug intoxication[J]. Resuscitation. 2012;83(11):14137. 
17. Johnson NJ, Gaieski DF, Allen SR, et al. A review of emergency cardiopulmonary bypass for severe poisoning by cardiotoxic drugs[J]. J Med Toxicol. 2013;9(1):54-60.

18. Rao P, Khalpey Z, Smith R, et al. Venoarterial Extracorporeal Membrane Oxygenation for Cardiogenic Shock and Cardiac Arrest[J]. Circ Heart Fail. 2018;11(9):e004905.

19. Chan CW, Wu IL, Lee CH, et al. Successful Extracorporeal Life Support in a Case of Severe Glyphosate-Surfactant Intoxication[J]. Crit Care Med. 2016;44(1):e45-7.

\section{Figures}



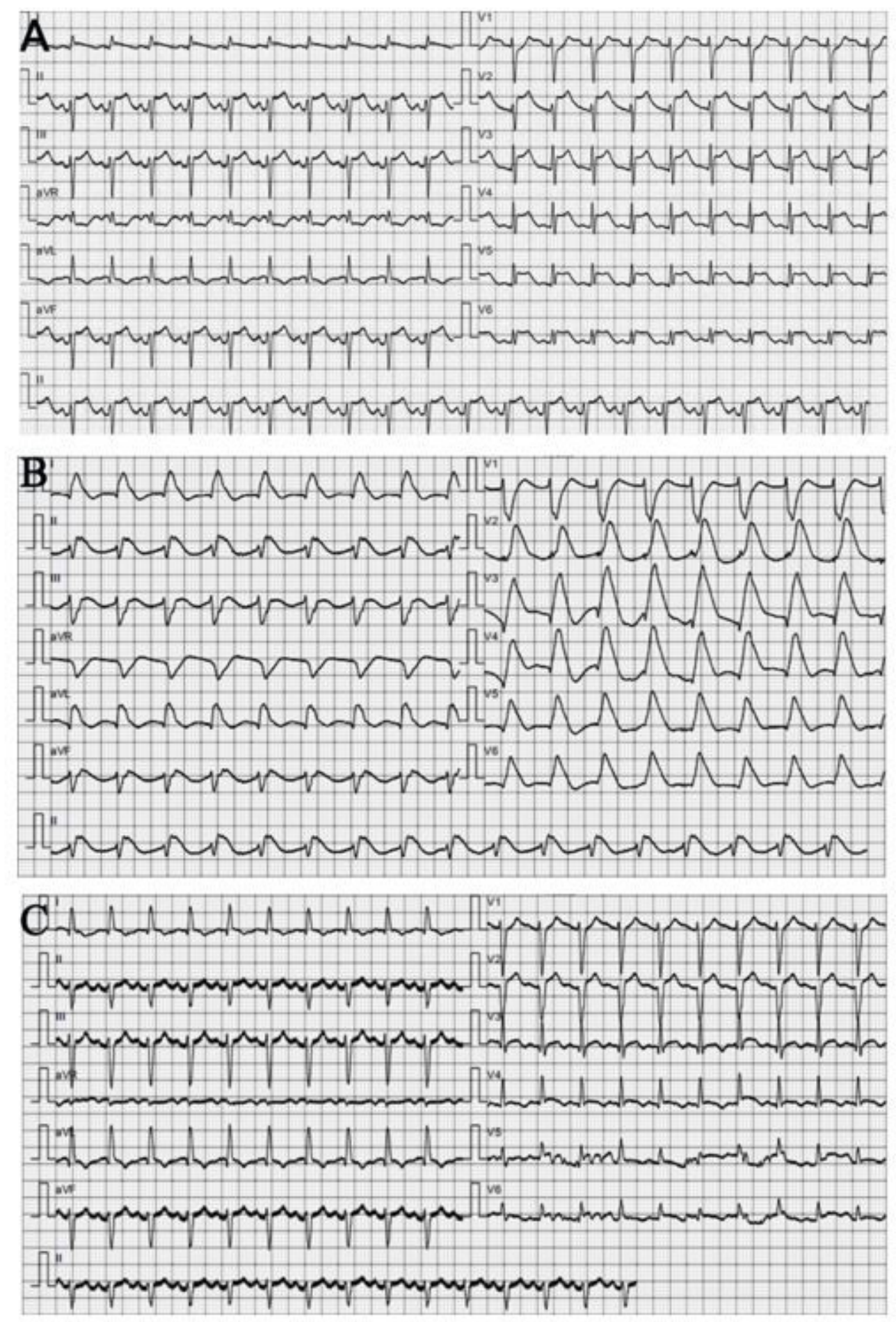

\section{Figure 1}

Panel A : Twenty-three hours after admission, the ECG showed extensive lead ST segment elevation. Panel B: The emergency ECG revealed ventricular arrhythmia 34 hours after admission. Panel C: The ECG showed a decrease in the ST segment 3 hours after VA-ECMO was performed. 